\title{
Axillary dissection in primary breast cancer: variations of the surgical technique and influence on morbidity
}

This article was published in the following Dove Press journal:

Cancer Management and Research

26 April 2012

Number of times this article has been viewed

\section{Sebastian Wojcinski ${ }^{1, *}$ \\ Sirin Nuengsri ${ }^{2}$,* \\ Peter Hillemanns ${ }^{3}$ \\ Werner Schmidt ${ }^{4}$ \\ Mustafa Deryal ${ }^{5}$ \\ Kubilay Ertan ${ }^{6}$ \\ Friedrich Degenhardt ${ }^{1}$}

'Franziskus Hospital, Department of Obstetrics/Gynecology, Bielefeld,

Germany; ${ }^{2}$ Karlsruhe Hospital, Department of Internal Medicine, Karlsruhe, Germany; ${ }^{3}$ Hannover Medical School, Department of Obstetrics/Gynecology, Hannover, Germany; ${ }^{4}$ University Hospital of

Saarland, Department of Obstetrics/ Gynecology, Homburg/Saar, Germany; ${ }^{5}$ Caritas Hospital Saarbrücken St Theresia, Department of Obstetrics/Gynecology, Saarbrücken, Germany; 'Leverkusen Hospital, Department of Obstetrics/ Gynecology, Leverkusen, Germany

*The first two authors contributed equally to this manuscript
Correspondence: Sebastian Wojcinski Franziskus Hospital, Department of Obstetrics/Gynecology, Kisker Str 27, 33615 Bielefeld, Germany

Tel +4952I 589 I50I;

+4952I 58971505

Fax +4952I 5891504

Email s@wojcinski.de

\begin{abstract}
Lymphedema of the arm is the most common and impairing complication after breast cancer surgery with axillary lymph node dissection (ALND). Our prospective study evaluated the effect of two different surgical techniques for ALND on postoperative morbidity. Patients were scheduled to undergo ALND. Patients in group $1(n=17)$ underwent the most common and standard technique of ALND, which uses sharp dissection of the tissue and subsequent electrocoagulation of bleedings. Patients in group $2(n=17)$ underwent a modified standard technique of ALND with clamping and ligatures of all resection margins. Postoperative wound secretion was quantified and patients were followed up for 6 months to assess long-term morbidity. The variations in surgical technique had no significant influence on the outcome variables. However, patients in group 2 showed a tendency to less wound secretion $(713$ versus $802 \mathrm{~mL}$; $P=$ nonsignificant $)$, a decreased rate of immediate postoperative seromas ( 11.8 versus $23.5 \%$; $P=$ nonsignificant $)$ and less lymphedema after 3 months (29.4 versus $41.2 \% ; P=$ nonsignificant). Moreover, the number of resected lymph nodes correlated with the total amount of drained fluid ( $P=0.006)$, the duration of the drain $(P=0.015)$, and the risk for the development of lymphedema after 3 months $(P=0.016)$. The described variations in surgical technique had no influence on the outcomes of the patients. The number of resected axillary lymph nodes remains the most important risk factor for treatment-related morbidity. Therefore, a well-balanced choice of the extent of the axillary dissection should be the surgeon's main concern.
\end{abstract}

Keywords: breast cancer, axillary dissection, lymphedema, morbidity

Lymphedema of the arm is a major complication that women face after breast cancer surgery with axillary lymph node dissection (ALND). The literature describes an incidence between $2 \%$ and $51 \%$, based on either subjective or objective findings. ${ }^{1-4}$ The development of lymphedema depends on treatment-related factors, such as the radicalness of surgery. Other known risk factors are adjuvant systemic chemotherapy or adjuvant radiation (and the combination of both), wound infections, the number of metastatic lymph nodes (LNs), obesity, hypertension, the patient's age at diagnosis, and excessive use of the limb after surgery. ${ }^{5}$

Today, sentinel node biopsy (SNB) is the standard therapy for women that are preoperatively staged with a negative nodal status. ${ }^{6}$ The precision of histological staging by SNB is very high, and it significantly minimizes postoperative morbidity. ${ }^{5}$ If axillary metastases are suspected in the clinical examination, or if there was neoadjuvant therapy, the success of SNB may be impaired. These patients should still undergo conventional ALND, which is a more extensive intervention compared with SNB. In these cases, it is recommended to explore level I (from the lateral side of the 
small pectoralis muscle over the thoracodorsal nerve and vessels to the lateral chest wall) and level II (resection of the fatty tissue of the axilla and underneath the pectoralis minor muscle). The aim of ALND is to remove all clinically apparent manifestations of the disease and to dissect at least ten lymph nodes for pathologic evaluation to stage the axilla accurately. Abundant surgery beyond level I and II nodes should be avoided, and axillary dissection is extended to level III nodes only if gross disease is apparent in level I or II nodes or if there are clinically suspicious lymph nodes in level III nodes. ${ }^{7}$ The traditional ALND procedure implies a significant increase in morbidity, such as lymphedema or paresthesia of the arm. ${ }^{8}$

Regarding the surgical technique of ALND, the tissue is usually resected using a scalpel or either conventional or bipolar scissors, or it is gently disrupted using other instruments. Larger blood vessels are either clamped and ligated or coagulated. However, small lymphatic vessels and capillary blood vessels are not completely sealed. Alternatively, in order to close these small vessels efficiently, tissue bridges can be dissected by clamping and ligation (ie, sutures).

The objective of our study was to compare two different standard techniques for ALND that involved either cutting and coagulation or clamping and ligation with respect to postoperative morbidity. We hypothesized that the consequent occlusion of small vessels with ligatures may have clinical benefits for the patient.

\section{Methods and materials Study design}

Our study was carried out between January 2007 and October 2008 at the Breast Cancer Center of the University Hospital of Saarland, Homburg/Saar, Germany.

Two different standard surgical techniques for ALND were compared in group 1 and group 2, respectively.

In group 1, the tissue was dissected with conventional scissors or gently disrupted with other instruments. The vessels were coagulated whenever bleeding occurred, and only large vessels were clamped and ligated. As a matter of course, the relevant anatomical structures (ie, the thoracodorsal nerve and thoracodorsal vessels, the long thoracic nerve, the intercostobrachial nerves, and the axillary vein) were thoroughly identified and preserved. In this technique, small lymphatic vessels and capillary blood vessels are not completely sealed. This surgical technique is the most commonly used in ALND. ${ }^{9}$

In group 2, the axillary tissue was carefully explored without cutting or disrupting the lymphatic vessels, blood vessels, or connective tissue structures. The fatty tissue was gently mobilized and separated from more solid connective tissues and relevant anatomical structures (see above). The remaining tissue bridges were then clamped, cut through with conventional scissors, and ligated. For the ligature, we used a midterm braided and coated synthetic absorbable material (Safil ${ }^{\circledR}$ Violet 3/0; B Braun Melsungen AG, Inc, Melsungen, Germany). This procedure was intended to occlude even small lymphatic vessels and capillary blood vessels.

Our study cohort was recruited from patients who attended our breast cancer center seeking management of their disease. All patients were diagnosed and treated according to standard protocols. ${ }^{7,10}$ Patients with invasive breast cancer who were scheduled to undergo ALND due to clinically suspicious lymph nodes were regarded as suitable for our study. Patients with a history of breast or axilla surgery, orthopedic problems in the arm or shoulder joint, inflammatory conditions of the breast, or skin disorders were excluded. Patients who met the inclusion criteria and who agreed to be enrolled in our study were randomized to be treated either in group 1 (ALND based on cutting and coagulation, $\mathrm{n}=17$ ) or in group 2 (ALND based on clamping and ligation, $n=17$ ). The surgical procedures were performed by the authors KE and MD.

As both techniques for ALND represent standard surgical techniques, the responsible ethics committee did not require additional approval for our study design. All patients gave their written, informed consent for the selected surgical procedure.

\section{Postoperative assessment and study endpoint}

During the surgical procedure, all patients received a 12 gauge suction drain (Redon type). ${ }^{11}$

The primary study objective was to measure the amount of postoperative wound secretion on a daily basis. In addition, data was generated concerning the composition of the drained fluid (protein content and hemoglobin) 24 hours after surgery. Postoperative treatments, such as dressings, infusions, mobilization, and physical therapy were carried out identically in both groups. The drain was removed as soon as the amount of secretion was $\leq 30 \mathrm{~mL}$ within 24 hours or $\leq 50 \mathrm{~mL}$ within 24 hours on two consecutive days.

The secondary study objective was to determine the rate of lymphedema after 3 and 6 months. As a matter of fact, none of the existing rating scales for lymphedema have been formally validated. ${ }^{12}$ Our study defined arm lymphedema as a difference in the circumference of the 
arms of at least $4 \mathrm{~cm}$ at the point of greatest visible difference. It included subjective complaints or the necessity for medical treatment, such as manual compression lymphatic massage, compression garments, or bandaging. This definition corresponds to a lymphedema grade 2 or higher according to the late effects toxicity scoring system of the EORTC. ${ }^{13}$

Adjuvant treatment was carried out according to the common standards: All patients who were treated with breastconserving surgery received adjuvant radiotherapy of the breast. None of the patients received axillary radiotherapy. Adjuvant chemotherapy was applied whenever it was indicated. The route of the administration of chemotherapy was either intravenous in the contralateral arm or in an implanted port on the contralateral side.

\section{Statistical analysis}

Excel $^{\circledR} 2007$ (Microsoft Corporation, Redmond, WA) was used for data collection. Statistical analysis was performed by the author SN and validated by SW. The analysis was performed with MedCalc ${ }^{\circledR} 11.6$ statistical software (MedCalc Software bvba, Mariakerke, Belgium).

Results are presented as the mean \pm standard deviation (SD), counts, and/or percentages with $95 \%$ confidence intervals $(95 \% \mathrm{CI})$ where applicable. A Student's $t$-test was used for comparison of means for the numerical data. Categorical data were compared using Fisher's exact test for univariate distributions and Yates's chi-square test for multivariate distributions. Odds ratios (OR) were used to explore risk factors further for postoperative morbidity. For correlations, we applied Pearson's product moment correlation. Statistical significance was assumed as $P<0.05$ for all tests.

\section{Results}

All patients were treated according to the randomized study protocol, and there were no protocol violations. None of the patients dropped out, and all patients were available for the planned follow-up after 3 and 6 months. Therefore, all 34 patients were included in the analysis.

Concerning the demographic data, the tumor stage, and the details about the surgical procedure, there were no significant differences between the groups. The detailed results are shown in Table 1.

After surgery, the total amount of drained fluid was $802 \pm 546 \mathrm{~mL}$ in group 1 and $713 \pm 382 \mathrm{~mL}$ in group 2. Despite the tendency, the difference was not significant $(P=0.58)$. Furthermore, regarding the daily discharge of fluid over time, the course of both curves was nearly identical (Figure 1). Consequently, the duration of the suction drain was identical in both groups with $10.1 \pm 4.0$ days in group 1 and $9.5 \pm 3.9$ days in group $2(P=0.64)$.

When comparing the protein and hemoglobin content of the drained fluid 24 hours after surgery, we found a protein concentration of $41.3 \pm 7.3 \mathrm{~g} / \mathrm{L}$ in group 1 and $43.2 \pm 8.1 \mathrm{~g} / \mathrm{L}$ in group 2. Concerning hemoglobin levels, the content was $2.4 \pm 1.3 \mathrm{~g} / \mathrm{dL}$ in group 1 and $2.7 \pm 1.8 \mathrm{~g} / \mathrm{dL}$ in group 2 . These differences were not significant $(P=0.48$ and $P=0.57$, respectively).

There was a tendency for more postoperative seroma requiring interventions in group $1(23.5 \%)$ compared with group 2 (11.8\%). The odds ratio for this complication was 2.31 (95\% CI: 0.36-14.72) and failed to be statistically significant $(P=0.38)$. We also found a tendency for more lymphedema after three months in group 1 (41.2\%) compared

Table I Group comparison (mean \pm standard deviation, range, or rate)

\begin{tabular}{|c|c|c|}
\hline & $\begin{array}{l}\text { Group I } \\
\text { (cutting and coagulation) } \\
(n=I 7)\end{array}$ & $\begin{array}{l}\text { Group } 2 \\
\text { (clamping and ligation) } \\
(n=17)\end{array}$ \\
\hline Age (years) & $59.4 \pm 12.4(45-84)$ & $60.3 \pm 12.1(39-83)$ \\
\hline BMI $\left(\mathrm{kg} / \mathrm{m}^{2}\right)$ & $25.0 \pm 2.0(21-40)$ & $28.2 \pm 5.2(19-39)$ \\
\hline Blood pressure preoperative (systolic/diastolic, mmHg) & $129 \pm 14 / 76 \pm 11(100-170 / 55-95)$ & $127 \pm 16 / 75 \pm 11(80-155 / 60-100)$ \\
\hline $\mathrm{BCT}(\%, \mathrm{n})$ & 64.7\% (II/I7) & $64.7 \%(11 / 17)$ \\
\hline Total duration of surgery (min) & $117 \pm 36$ & $127 \pm 54$ \\
\hline PTI $(\%, n)$ & $35.3 \%(6 / 17)$ & $52.9 \%(9 / 17)$ \\
\hline pT2 $(\%, n)$ & $58.8 \%(10 / 17)$ & $41.2 \%(7 / 17)$ \\
\hline pT3/pT4 (\%, n) & $5.9 \%(1 / 17)$ & $5.9 \%(1 / 17)$ \\
\hline CT $(\%, n)$ & $76.5 \%(13 / 17)$ & $76.5 \%(13 / 17)$ \\
\hline RT if BCT $(\%, n)$ & $100 \%(1 \mathrm{I} / \mathrm{II})$ & $100 \%(I I / I I)$ \\
\hline PMRT $(\%, n)$ & $33.3 \%(2 / 6)$ & $33.3 \%(2 / 6)$ \\
\hline $\mathrm{ET}$ if $\mathrm{HR}+(\%, \mathrm{n})$ & $100 \%(13 / 13)$ & $100 \%(14 / 14)$ \\
\hline
\end{tabular}

Note: Differences between group I and group 2 are statistically not significant.

Abbreviations: BCT, breast-conserving therapy; CT, chemotherapy; ET, endocrine therapy; HR+, hormone responsive; PMRT, postmastectomy radiation therapy; PT, pathologic tumor stage; RT, radiation therapy. 


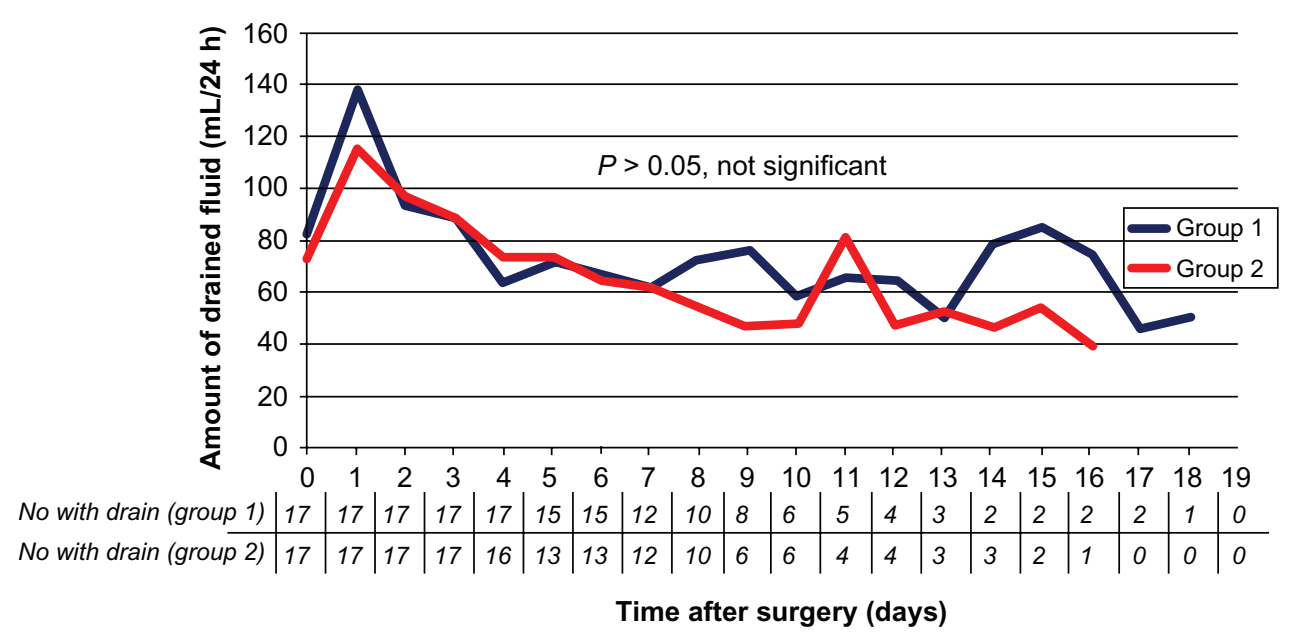

Figure I Comparison of the amount of drained fluid drained from the axilla between group I (surgery based on cutting and coagulation) and group 2 (surgery based on clamping and ligatures).

Note: The difference between the groups was not statistically significant.

with group 2 (29.4\%). This difference was not significant either $(\mathrm{OR}=1.68,95 \% \mathrm{CI}$ : 0.41-6.96; $P=0.47)$. After six months of observation, there was no difference regarding the rate of arm lymphedema in both groups ( $47.1 \%$ versus $47.1 \%$, respectively; $P=1.00$ ). No other complications beyond seroma and/or arm lymphedema occurred in the two groups (eg, infections, reduction of the range of motion, chronic pain). The results are summarized in Table 2.

Because the variations in surgical technique showed only a nonsignificant influence on morbidity, we further explored the known, classical risk factors in our study collective $(n=34)$ and focused on the number of resected
LNs as an indicator of the radicalness of the surgical procedure.

There was a strong correlation between the number of resected LNs, the total amount of drained fluid $(\mathrm{r}=0.45$; $P=0.006)$, and the duration of the suction drain $(\mathrm{r}=0.41$; $P=0.015)$. Both parameters significantly increased according to the number of resected LNs (Figure 2).

Regarding the patients who suffered from seroma of the axilla after removal of the drain, there was no difference in the number of resected LNs ( 12.0 versus $11.7 \mathrm{LNs} ; P=0.92$ ). On the other hand, patients who developed arm lymphedema after 3 or 6 months had a higher number of resected LNs

Table 2 Comparison of the outcome variables between group I and group 2 (mean \pm standard deviation, range, or rate)

\begin{tabular}{|c|c|c|}
\hline & $\begin{array}{l}\text { Group I } \\
\text { (cutting and coagulation) } \\
(n=I 7)\end{array}$ & $\begin{array}{l}\text { Group } 2 \\
\text { (clamping and ligation) } \\
(n=17)\end{array}$ \\
\hline Total number of resected lymph nodes (n) & $\mathrm{II} . \mathrm{I} \pm 6.4$ & $13.6 \pm 5.7$ \\
\hline Number of resected lymph nodes level I (n) & $6.8 \pm 4.5$ & $9.8 \pm 4.1$ \\
\hline Number of resected lymph nodes level II (n) & $3.4 \pm 2.5$ & $3.0 \pm 2.8$ \\
\hline Number of resected lymph nodes level III (n) & $0.9 \pm 1.7$ & $0.8 \pm 1.0$ \\
\hline \multicolumn{3}{|l|}{ Primary outcome variables } \\
\hline Total amount of drained fluid $(\mathrm{mL})$ & $803 \pm 546(160-1480)$ & $712 \pm 382(110-2270)$ \\
\hline Duration of suction drain (days) & $10.1 \pm 4.0(5-14)$ & $9.5 \pm 3.9(4-19)$ \\
\hline Immediate seroma after removal of suction drain (\%) & 23.5 & 11.8 \\
\hline \multicolumn{3}{|l|}{ Secondary outcome variables } \\
\hline Lymphedema after 3 months $(\%, n)$ & $4 I .2(7 / 17)$ & $29.4(5 / 17)$ \\
\hline Lymphedema after 6 months $(\%, \mathrm{n})$ & $47.1(8 / 17)$ & $47.1(8 / 17)$ \\
\hline Other morbidity concerning the axillary region & $0(0 / 17)$ & $0(0 / 17)$ \\
\hline \multicolumn{3}{|l|}{ after 3 and 6 months $(\%, n)$} \\
\hline \multicolumn{3}{|l|}{ Additional data } \\
\hline Total protein content of drained fluid $(\mathrm{g} / \mathrm{L})$ & $41.3 \pm 7.3(25-52)$ & $43.2 \pm 8.1(30-59)$ \\
\hline Hemoglobin content of drained fluid $(\mathrm{g} / \mathrm{dL})$ & $2.4 \pm 1.3(0.3-4.6)$ & $2.7 \pm 1.8(0.5-6.1)$ \\
\hline
\end{tabular}

Note: Differences between group I and group 2 are statistically not significant. 
A

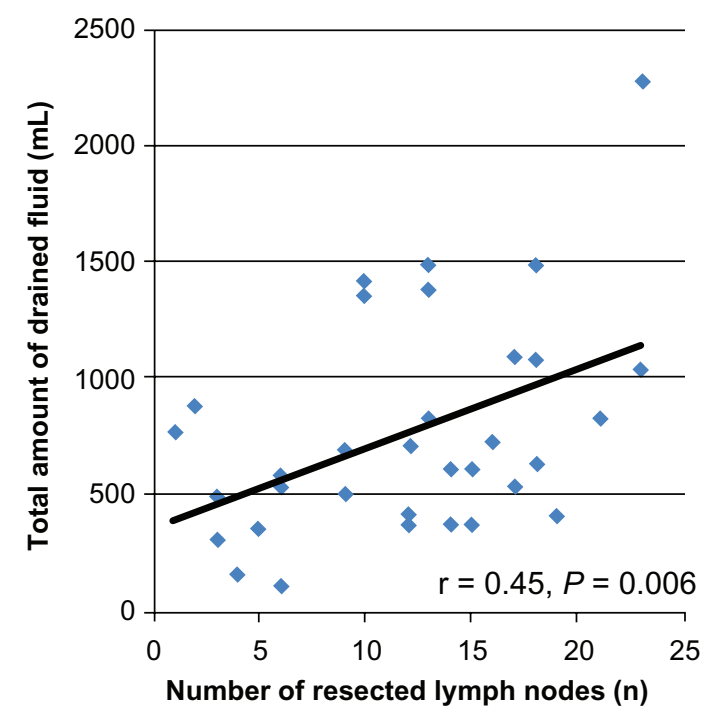

B

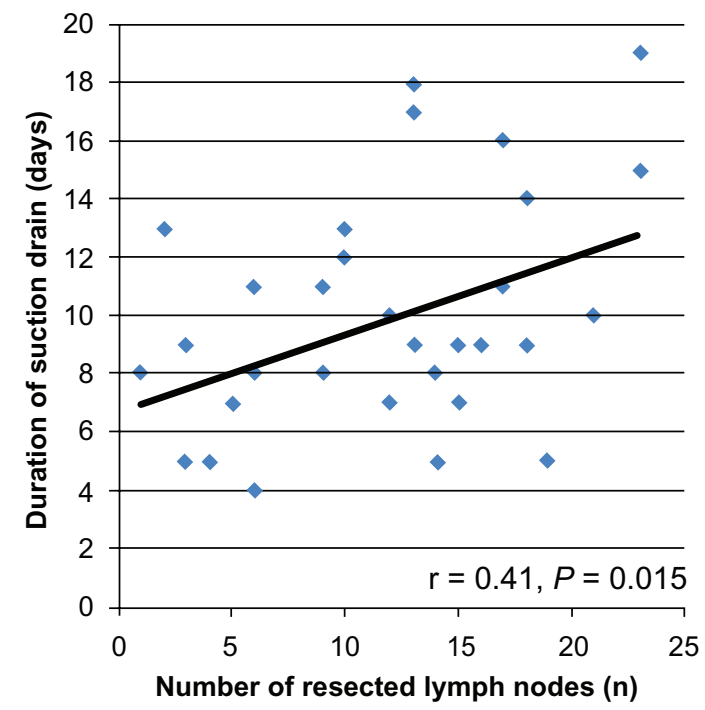

Figure 2 Positive correlation between the number of resected $L N s$ and the total amount of fluid drained from the axilla $(P=0.006 ; \mathbf{A})$ and a positive correlation between the number of resected $L N$ s and the duration of the drain $(P=0.015 ; \mathbf{B})$.

Abbreviation: LNs, lymph nodes.

compared with patients who did not experience lymphatic problems (after 3 months 15.2 versus $9.9 \mathrm{LNs} ; P=0.016$; after 6 months 13.9 versus $9.8 \mathrm{LNs} ; P=0.054$, trend towards statistical significance).

Because both the protein concentration and hemoglobin concentration increased with an increasing number of resected LNs, there was also a correlation between the number of resected LNs and the quality of the drained fluid $(\mathrm{r}=0.33$; $P=0.054$, trend and $\mathrm{r}=0.36 ; P=0.045$ ) (Figure 3). On the other hand, the composition of the fluid was not suitable as a predictor of the development of arm lymphedema. In both patient groups (those with and those without lymphedema), the distribution of protein and hemoglobin in the fluid was not different (for protein 42.9 versus $41.7 \mathrm{~g} / \mathrm{L} ; P=0.68$; for hemoglobin 2.56 versus $2.43 \mathrm{~g} / \mathrm{dL} ; P=0.53$, trend).

\section{Discussion}

Our study attempted to determine whether immediate postoperative and long-term morbidity after axillary dissection could be reduced by variations in surgical technique.

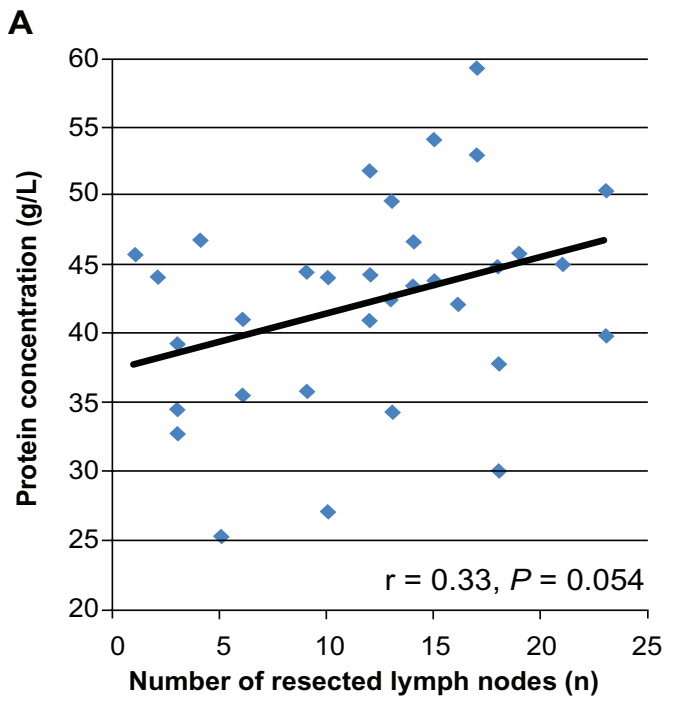

B

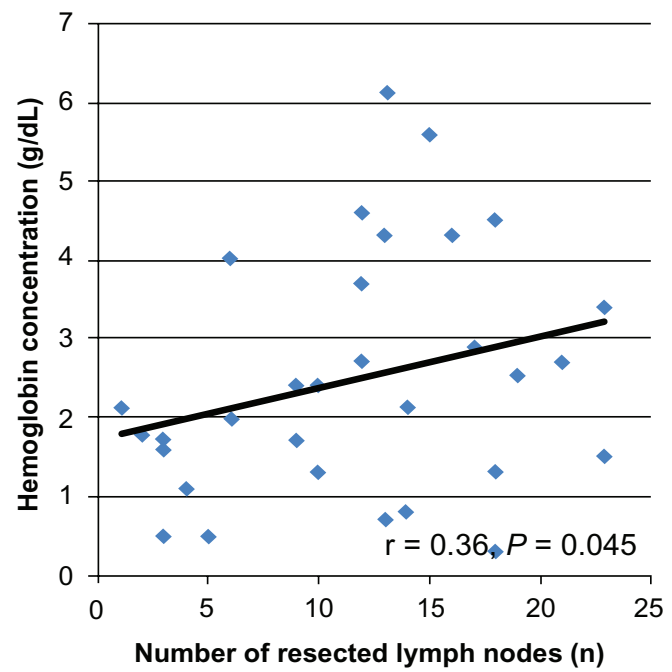

Figure 3 Positive correlation between the number of resected LNs and the protein concentration in the drained fluid $(P=0.054 ; \mathbf{A})$ and a positive correlation between the number of resected $L N s$ and hemoglobin in the drained fluid $(P=0.045 ; \mathbf{B})$. Abbreviation: LNs, lymph nodes. 
Although SNB constitutes a standard procedure in breast cancer, women with clinically suspicious LNs or histologically confirmed metastases cannot benefit from this gentle procedure. ${ }^{6}$ However, these women may profit from a less aggressive surgical technique for ALND in terms of reduced morbidity without impairment of the oncological outcome.

Concerning the relevant parameters, throughout the follow-up time of 6 months, we found no statistically significant differences related to the surgical technique used. Patients who need to undergo ALND for histologically proven or suspected metastatic LNs must still accept a high risk of arm lymphedema, which was a maximum of $47.1 \%$ in our study. Generally, the rate of arm lymphedema in our study was quite high, although it was within the reported range quoted in the literature, which describes arm lymphedema in $2 \%$ to $51 \%$ of patients. ${ }^{2-4}$ This high rate might be due to the focus on patients with clinically suspicious lymph nodes in addition to the relatively strict definition of arm lymphedema applied in our study. However, because of the high rate of lymphedema, other techniques of ALND and combinations of different techniques should be further investigated.

The technique that was applied to the patients in group 2 results in a higher amount of foreign material left in the body. On the other hand, the electrocoagulation technique used in group 1 causes more thermal trauma to the tissue. Comparison of these two types of tissue irritation in our study showed that the total amount of fluid was lower in group 2 than in group 1. The rate of immediate postoperative seroma was also reduced although the differences were statistically not significant. However, there were slightly more pT2 tumors in group 1 (58.5\% versus $41.2 \%$, not significant) which might influence this outcome variable because of the more extensive surgery to the breast. On the other hand, this result might suggest a minor influence of the surgical technique on recovery after ALND although we were not able to prove this effect in our study.

In 2006, Agrawal et al demonstrated that electrocautery dissection causes thermal trauma and results in increased volumes of seroma. ${ }^{14}$ Therefore, the technique of clamping and ligature is an opportunity to reduce thermal trauma of the tissue without presenting known disadvantages.

In 1999, Bonnema et al investigated the composition of the axillary fluid discharge after ALND. The authors showed that on the first day after ALND, the serous fluid contained higher amounts of blood particles and then changed to a lymphatic-like fluid. ${ }^{15}$ In our study, we did not repeat the analysis of the fluid over time, but assessed the fluid 24 hours after surgery and correlated the results with the surgical technique and the risk for the development of lymphedema.
There was no correlation in this regard. All patients had a similar composition of the fluid.

Interestingly, we found a correlation between the radicalness of the surgery, expressed as the number of resected LNs, and the amount of blood particles and protein in the fluid. The hemoglobin content rose with more extensive ALND, which can be explained by more bleeding related to the larger region that was surgically explored. The protein content of the drained fluid averaged about $40 \mathrm{~g} / \mathrm{L}$, fulfilling the definition of an exudation. Exudates are usually caused by tissue trauma or inflammation, whereas transudates are a consequence of increased hydrostatic pressure or decreased colloid oncotic pressure in the vessels. Therefore, the tissue trauma associated with more resected LNs may result in an increased protein content of the wound secretion, as we demonstrated in our study. Although these effects are significant and have plausible physiological explanations, the analysis of the wound secretion does not permit risk prediction for the development of lymphedema.

Regarding our entire study population, we found a significant correlation between the number of resected LNs and the total amount of drained fluid, resulting in a significant effect on the duration of wound drainage. Furthermore, the number of resected LNs had an impact on the subsequent development of arm lymphedemas although this difference only showed a trend towards statistical significance, which may be due to the small sample size. After surgery, there are fewer lymph nodes and lymphatic vessels to process the lymphatic fluid. Consequently, lymphatic fluid may accumulate and cause swelling along the arm. If just a few nodes are removed (ie, SNB), the risk for lymphedema is lower and may be a temporary condition, but if most lymph nodes are removed (ie, ALND), lymphedema is more likely and may be a permanent problem. With respect to this important result, the variations in the surgical technique had no effect on the morbidity of the patients in our study.

The main limitation of our study is the relatively small sample size $(\mathrm{n}=34)$, with only 17 probands in each group. Therefore, our study is unable to answer fully the question of an optimal surgical technique. If we used our primary outcome variables to calculate the necessary sample size in order to achieve a power of 0.80 with a type I error rate of 0.05 , a subsequent study using ten times the number of patients would be required.

Nevertheless, our results suggest that the variations in surgical technique have only limited influence on the immediate postoperative and long-term morbidity of the patient and that the radicalness of the surgery, reflected in 
the number of resected LNs, remains the main risk factor as reported previously. ${ }^{16}$

Another limitation is the short follow-up period of 6 months. Three months after surgery, the rate of arm lymphedema was $41.2 \%$ in group 1 and $29.4 \%$ in group 2 . After 6 months, both groups exhibited the same rate of lymphedema (47.1\%). Furthermore, arm lymphedema is known to occur occasionally, even many years after surgery. Lymphedema is a complex side effect of breast cancer treatment and has a crucial impact on the patient's quality of life. Patients report pain, increased circumference, and limited function of the arm. Furthermore, there is an increased risk for subsequent severe infections. Finally, lymphedema also impairs psychovegetative health. ${ }^{17}$ Therefore, an even longer follow-up would be necessary to detect all lymphedemas in both groups precisely. Nevertheless, in current studies it is common to evaluate the rate of lymphedema only 6 months after surgery. ${ }^{1}$

\section{Conclusion}

Our results demonstrate that the number of resected axillary LNs remains the most important risk factor for treatmentrelated morbidity. The choice of surgical technique had no influence on the outcome of the patient. Therefore, a wellbalanced choice of surgical technique based on the extent of the axillary dissection and oncological considerations should be the main concern of the surgeon.

\section{Authors' contributions}

SW and WS contributed to the conception and design of the study. MD and KE performed the surgical procedures according to our study protocol. SN contributed to data collection. SW and SN contributed to the statistical analysis and interpretation of the data. SN and SW contributed to writing and reviewing the manuscript. FD and $\mathrm{PH}$ conducted final reviews of the database and the manuscript and WS provided methodological advice. SW, WS, MD, KE, and SN were employees at the University Hospital of Saarland at the time of the study.

\section{Disclosure}

The authors report no conflicts of interest in this work.

\section{References}

1. Wang Z, Wu LC, Chen JQ. Sentinel lymph node biopsy compared with axillary lymph node dissection in early breast cancer: a meta-analysis. Breast Cancer Res Treat. 2011;129:675-689.

2. Schijven MP, Vingerhoets AJ, Rutten HJ, et al. Comparison of morbidity between axillary lymph node dissection and sentinel node biopsy. Eur J Surg Oncol. 2003;29:341-350.

3. Mathew J, Barthelmes L, Neminathan S, Crawford D. Comparative study of lymphedema with axillary node dissection versus axillary node sampling with radiotherapy in patients undergoing breast conservation surgery. Eur J Surg Oncol. 2006;32:729-732.

4. Kahtcheressian JL, Wolff AC, Smith TJ, et al. American Society of Clinical Oncology 2006 update of the breast cancer follow-up and management guidelines in the adjuvant setting. $J$ Clin Oncol. 2006;24:5091-5097.

5. Kocak Z, Overgaard J. Risk factors of arm lymphedema in breast cancer patients. Acta Oncol. 2000;39:389-392.

6. Kuehn T, Bembenek A, Decker T, et al. A concept for the clinical implementation of sentinel lymph node biopsy in patients with breast carcinoma with special regard to quality assurance. Cancer. 2005;103:451-461.

7. National Comprehensive Cancer Network, Inc. The NCCN Clinical Practice Guidelines in Oncology ${ }^{\mathrm{TM}}$ : Breast Cancer (V.2.2010). 2010. Available from: http://www.nccn.org/professionals/physician_gls/pdf/ breast.pdf. Accessed March 27, 2012.

8. Lucci A, McCall LM, Beitsch PD, et al. Surgical complications associated with sentinel lymph node dissection (SLND) plus axillary lymph node dissection compared with SLND alone in the American College of Surgeons Oncology Group Trial Z0011. J Clin Oncol. 2007;25:3657-3663.

9. Zuber M, Oertli D, Marti WR, et al. Guideline for axillary dissection. Schweizerische Aerztezeitung. 2003;84:1967-1973.

10. Breast Commission of the German Gynecological Oncology Working Group (AGO). Diagnosis and Treatment of Patients with Primary and Metastatic Breast Cancer. Recommendations 2010. Available from: http://www.ago-online.org/_download/unprotected/g mamma_10_1_1_00_introduction.pdf. Accessed on March 27, 2012.

11. Redon H, Jost A, Troques A. Closure under reduced atmospheric pressure of extensive wounds. Mem Acad Chir. 1954;80:394-396.

12. Cheville AL, McGarvey CL, Petrek JA, Russo SA, Thiadens SR, Taylor ME. The grading of lymphedema in oncology clinical trials. Semin Radiat Oncol. 2003;13:214-225.

13. Pavy JJ, Denekamp J, Letschert J, et al; EORTC Late Effects Working Group. Late effects toxicity scoring: the SOMA scale. Radiother Oncol. 1995;35:11-15.

14. Agrawal A, Ayatunde AA, Cheung KL. Concepts of seroma formation and prevention in breast cancer surgery. ANZ J Surg. 2006;76: 1088-1095.

15. Bonnema J, Ligtenstein DA, Wiggers T, van Geel AN. The composition of serous fluid after axillary dissection. Eur J Surg. 1999;165:9-13.

16. Tsai RJ, Dennis LK, Lynch CF, Snetselaar LG, Zamba GK, Scott-Conner C. The risk of developing arm lymphedema among breast cancer survivors: a meta-analysis of treatment factors. Ann Surg Oncol. 2009;16:1959-1972.

17. Erickson VS, Pearson ML, Ganz PA, Adams J, Kahn KL. Arm edema in breast cancer patients. J Natl Cancer Inst. 2001;93:96-111.
Cancer Management and Research

\section{Publish your work in this journal}

Cancer Management and Research is an international, peer-reviewed open access journal focusing on cancer research and the optimal use of preventative and integrated treatment interventions to achieve improved outcomes, enhanced survival and quality of life for the cancer patient The journal welcomes original research, clinical \& epidemiological

\section{Dovepress}

studies, reviews \& evaluations, guidelines, expert opinion \& commentary, case reports \& extended reports. The manuscript management system is completely online and includes a very quick and fair peerreview system, which is all easy to use. Visit http://www.dovepress.com/ testimonials.php to read real quotes from published authors. 